\title{
Effect of seminal plasma on hipoosmotic swelling test in fresh alpaca spermatozoa
}

\begin{abstract}
Summary
A study was designed with the objective of evaluating the effect of seminal plasma on the response to the hypoosmotic swelling test (HOST) in alpaca spermatozoa, for which three experimental groups were organized as follows: Group 1(n=15) plasma free sperm seminal (obtained from the vas deferens, aspirated in PBS), Group 2(n=15) free seminal plasma sperm reconstituted with seminal plasma (obtained from the vas deferens, aspirated in PBS, mixed in 50/50\% with seminal plasma) and Group $3(\mathrm{n}=15)$ whole semen (obtained by artificial vagina), The samples were incubated in a hypoosmotic solution adjusted to $100 \mathrm{mOsmol}$ (sodium citrate + fructose $+2 \mathrm{H}_{2} \mathrm{Ocsp}$ $100 \mathrm{~mL}$ ). $0.1 \mathrm{~mL}$ of semen $+0.9 \mathrm{~mL}$ of hypoosmotic solution was mixed, incubated for 30 minutes in a water bath at $37^{\circ} \mathrm{C}$ and the reaction was stopped with $0.1 \mathrm{~mL}$ of $4 \%$ formaldehyde. A count of at least 200 spermatozoa was performed per sample, using an optical microscope with immersion objective (100X), the vitality was evaluated by supravital eosin staining $(0.7 \%)$-nigrosin $(1 \%)$, the results indicate that it does not exist a detrimental effect of the seminal plasma on the endosmotic response, being, on the contrary, superior in the whole semen; the vitality of the spermatozoa with and without seminal plasma is similar, however it decreases when it is reconstituted with seminal plasma, possibly due to the seminal plasma of another animal; there is no positive correlation between endosmosis and vitality, indicating that the latter would not necessarily reflect the integrity of the membrane, which is why it is recommended to perform this test routinely in alpaca semen exams.
\end{abstract}

Keywords: endosmosis, seminal plasma, spermatozoon, alpaca
Volume 2 Issue 4 - 2017

\author{
Pacheco Jl,' Mamani Cato RH, ${ }^{2}$ Vélez VM' \\ 'Department of Veterinary Medicine, National University of San \\ Marcos, Perú \\ ${ }^{2}$ National Institute of Agrarian Innovation (INIA), Perú
}

Correspondence: Pacheco Jl, Department of Veterinary Medicine- National University of San Marcos, Perú, Emailmvz_joelpc@hotmail.com

Received: December 04, 2017 | Published: December 22, 2017

\section{Introduction}

The process of fertilization can't be attributed only to the number of live, motile and normal sperm deposited within the female but especially to its functionality. ${ }^{1}$ The hypoosmotic test or hypoosmotic test (HOST) is a functional test, by which the live sperm are subjected to an incubation in a hypoosmotic solution, the sperm with the functional membrane will allow the entry of water by osmosis that will be evidenced by swelling and curling of the tail. ${ }^{2}$ In ejaculates of llamas obtained by artificial vagina, and subjected to said test, endosmosis percentages of $40 \%$ were observed using a solution of $100 \mathrm{mOsmol}^{3}{ }^{3}$ Other reports in flames indicate $33.48 \%$ in semen collected by electro ejaculation and $30.15 \%$ in semen collected by artificial vagina using hypoosmotic solution adjusted to $50 \mathrm{mOsmol} ;{ }^{4}$ and a last report indicates $36 \%$ in semen sperm collected by burning electro ejaculation using also hypoosmotic solution adjusted to $50 \mathrm{mOsmol} .5$ In alpaca ejaculates obtained by electro ejaculation, percentages of endosmosis were obtained with a range of 20 to $62 \%$ using 100 mosmol hypoosmotic solution, ${ }^{6}$ while in alpacas whole semen $23.5 \%$ was reported using hypoosmotic solution of $150 \mathrm{mOsmol}, 7$ Alpaca epididymal spermatozoa were subjected to the hypoosmotic test at an osmolarity of $100 \mathrm{mOsmol}$, obtaining a response of $89.08 \%$, evidencing a greater response in free sperm from seminal plasma. ${ }^{8}$ The artificial vagina method allows obtaining complete ejaculates of alpacas, ${ }^{9}$ free seminal plasma sperm can be obtained by means of the deferential vas deferens technique permanently, ${ }^{10}$ Under these considerations, the objective of the present investigation was to evaluate the endosmotic response of spermatozoa of alpaca in the presence and absence of seminal plasma and reconstituting the seminal plasma to sperm obtained from the vas deferens.

\section{Methodology}

Six male alpacas were used, three males donated whole semen using the artificial vagina technique, said semen was also used to obtain seminal plasma by centrifugation, at $2000 \mathrm{~g} / 20$ minutes and in three males free seminal plasma sperm were obtained, which were obtained by the deferential vas deferens technique. ${ }^{10}$ The sperm samples were divided into three groups per treatment, making a total of 45 samples: Group $1 \quad(n=15)$ free seminal plasma sperm (obtained from the vas deferens, aspirated in PBS), Group $2(n=15)$ free seminal plasma spermatozoa reconstituted with seminal plasma (obtained from the vas deferens, aspirated in PBS, mixed in 50/50\% with seminal plasma), and group $3(\mathrm{n}=15)$ whole semen (obtained by artificial vagina). The samples were incubated in a hypoosmotic solution adjusted to 100 mosmol (sodium citrate + fructose $+2 \mathrm{H}_{2} \mathrm{O}$ c.s.p. $100 \mathrm{~mL}$ ). $0.1 \mathrm{~mL}$ of semen $+0.9 \mathrm{~mL}$ of hypoosmotic solution was mixed, incubated for 30 minutes in a water bath at $37^{\circ} \mathrm{C}$ and the reaction was stopped with $0.1 \mathrm{~mL}$ of $4 \%$ formaldehyde. The count of sperm positive to endosmosis was performed, counting not less than 200 spermatozoa per sample using an optical microscope with immersion objective $(100 \mathrm{X})$, the vitality was evaluated by supravital eosin staining (0.7\%)-nigrosin (1\%). The statistical design used corresponded to a DCA, with previous transformation to the sine of the arc of the percentages of endosmosis and vitality influenced by each of the experimental groups, and Duncan's test to separate the average; also, the Pearson correlation test was used to relate the values of endosmosis and vitality; in both cases a probability value of $95 \%$ was used, the data were analyzed using the SAS V9.0 program. ${ }^{11}$ 


\section{Results and discussion}

The data on the effect of endosmosis and vitality influenced by the presence or absence of seminal plasma, are detailed in (Table 1). The endosmosis found in free seminal plasma sperm obtained from the vas deferens $(38.66 \%)$, is lower than the report of Rodríguez, ${ }^{8}$ who indicates $89.08 \%$ in epididymal sperm, possibly the sperm suffer changes in the permeability of its membrane during transit through the vas deferens. The spermatozoa that were reconstituted with seminal plasma presented a similar endosmotic response than those free of seminal plasma, which could be due to the amount of seminal plasma used, which corresponded to only $50 \%$ of the total volume prior to incubation, the decrease in vitality could be attributed to the use of seminal plasma from another animal; this finding is similar to the response reported by Carretero et al. ${ }^{5}$ who found $36 \%$ of endosmosis in flame semen obtained by electro ejaculation, where said semen is less viscous than the semen obtained by artificial vagina. The endosmosis of the whole semen with presence of seminal plasma was higher than groups 1 and 2, would indicate that the seminal plasma does not negatively influence this response as expected, being similar to the responses observed in llama spermatozoa in ejaculates obtained by vagina artificial, ${ }^{3}$ superior to those obtained by electroejaculation ${ }^{4}$ and similar to that described in semen of alpacas obtained by electroejaculation, ${ }^{6}$ and superior to what was obtained by Pacheco et al., ${ }^{7}$ who used a $150 \mathrm{mOsmol}$ hypoosmotic solution, while in the present study a 100 mosmol hypoosmotic solution was used. On the other hand, the correlations found between endosmosis and vitality were negative (less 0.2 group 1 , les 0.53 group 2 and 0.02 group 3), which indicates that there is an inversely proportional relationship, mainly in group 2, indicating that at lower vitality there is greater endosmosis, similar to that described by Pacheco et al., ${ }^{7}$ who indicate that there is no direct relationship between the two tests, which is why they recommend performing this test routinely since vitality does not necessarily indicate the functionality of the membrane of said sperm. There is no negative effect of seminal plasma on the response to the hypoosmotic swelling test in alpaca spermatozoa, with endosmosis in whole semen being higher; vitality is similar between sperm without seminal plasma and whole semen, however, it decreases when seminal plasma is added to free seminal plasma sperm; there is no positive correlation between endosmosis and vitality indicating that vitality does not necessarily reflect the integrity of the plasma membrane, so it is recommended to routinely perform this test on alpaca sperm.

Table I Alpaca sperm endosmosis in the presence and absence of seminal plasma

\begin{tabular}{llllllll}
\hline Espermatozoa & $\mathbf{n}$ & Host (\%) & D.S. & CV & Mín & Máx & Vitality (\%) \\
\hline Without Seminal Plasma & 15 & $38.66^{\mathrm{a}}$ & 7.82 & 20.07 & 26.19 & 59.49 & $69.12^{\mathrm{a}}$ \\
Recombinant with Seminal Plasma & 15 & $38.95^{\mathrm{a}}$ & 7.67 & 19.83 & 24 & 51 & $57.46^{\mathrm{b}}$ \\
With Seminal Plasma & 15 & $54.70^{\mathrm{b}}$ & 8.91 & 15.96 & 43 & 73 & $68.50^{\mathrm{a}}$ \\
\hline
\end{tabular}

\section{Acknowledgements}

None.

\section{Conflict of interest}

The author declares no conflict of interest.

\section{References}

1. Petrunkina AM, Waberski D, Günzel-Apel AR, et al. Determinants of sperm quality and fertility in domestic species. Reproduction. 2007;134(1):3-17.

2. Jeyendran RS, Van der Ven HH, Perez-Pelaez M, et al. Development of an assay to asses the functional integrity of the human sperm membrane and its relationships to other semen characteristics. J Reprod Fert. 1984;70(1):219-228.

3. Giuliano S, Ferrari MR, Spirito SE, et al. Advances in the implementation of the hypoosmotic test (Hos test) in llama spermatozoa. Argentina: 2007.

4. Giuliano S, Director A, Gambarotta M, et al. Collection method, season and individual variation on seminal characteristics in the llama (Lama glama). Anim Reprod Sci. 2008;104(3-4):359-369.
5. Carretero I, Giuliano S, Casaretto C, et al. Evaluación del ADN espermático de llamas utilizando azul de toluidina. Invet. 2009;11(1):5563.

6. Giuliano S, Casaretto C, Morán M, et al. Electroejaculation and seed characteristics of alpaca. DESCO center for studies and promotion of development Arequipa, Perú: III international camelids research symposium; 2010.

7. Pacheco JI, Deza HW, Mamani RH, et al. Evaluation of the hypoosmotic test response in fresh spermatozoa of alpaca. Abstracts of the XXXIV annual scientific meeting of the peruvian association of animal production. APPA Trujillo-Perú: 2011.

8. Rodriguez C. Effect of seminal plasma on the survival of cryopreserved spermatozoa of Alpaca Vicugna pacos. Peru: National University of San Marcos; 2009.

9. Bravo PW, Flores D, Ordoñez C. Effect of repeteated collection on semen characteristics of alpaca. Biol Reprod. 1997;57(3):520-524.

10. Perez GM, Zevallos, JP, Quintano J. Survival of alpaca sperm (Lama pacos) collected from the vas deferens in three diluters. Abstracts of the XVII National Congress of Veterinary Sciences, Peru: 2004.

11. SAS. User Installation guide for the SAS® system version 9 for Microsoft ${ }^{\circledR}$ Windows ${ }^{\circledR}$. USA: SAS Institute; 2002. p 1-15. 